\title{
Second-order magnetic critical points at finite magnetic fields: Revisiting Arrott plots
}

\author{
S. Bustingorry, ${ }^{1}$ F. Pomiro, ${ }^{2}$ G. Aurelio, ${ }^{1}$ and J. Curiale ${ }^{1}$ \\ ${ }^{1}$ CONICET, Centro Atómico Bariloche, 8400 San Carlos de Bariloche, Río Negro, Argentina \\ ${ }^{2}$ INFIQC (CONICET - Universidad Nacional de Córdoba), Departamento de Físicoquimica, Facultad de Ciencias Químicas, \\ Universidad Nacional de Córdoba, Haya de la Torre esq. Medina Allende, Ciudad Universitaria, X5000HUA Córdoba, Argentina
}

(Received 11 March 2016; revised manuscript received 6 June 2016; published 27 June 2016)

\begin{abstract}
The so-called Arrott plot, which consists in plotting $H / M$ against $M^{2}$, with $H$ the applied magnetic field and $M$ the magnetization, is used to extract valuable information in second-order magnetic phase transitions. Besides, it is widely accepted that a negative slope in the Arrott plot is indicative of a first-order magnetic transition. This is known as the Banerjee criterion. In consequence, the zero-field transition temperature $T^{*}$ is reported as the characteristic first-order transition temperature. By carefully analyzing the mean-field Landau model used for studying first-order magnetic transitions, we show in this work that $T^{*}$ corresponds in fact to a triple point where three first-order lines meet. More importantly, this analysis reveals the existence of two symmetrical second-order critical points at finite magnetic field $\left(T_{c}, \pm H_{c}\right)$. We then show that a modified Arrott plot can be used to obtain information about these second-order critical points. To support this idea we analyze experimental data on $\mathrm{La}_{2 / 3} \mathrm{Ca}_{1 / 3} \mathrm{MnO}_{3}$ and discuss an estimate for the location of the triple point and the second-order critical points.
\end{abstract}

DOI: 10.1103/PhysRevB.93.224429

\section{INTRODUCTION}

A full understanding of ferromagnetic materials necessarily comprises basic knowledge about the underlying paramagnetic-ferromagnetic phase transition. This refers to questions about the order of the magnetic transition, the value of the critical exponents, the behavior of the susceptibility and entropy changes, etc. Among the possible questions the most fundamental one could be what is the order of the phase transition.

Classical textbooks [1-3] teach that the paramagneticferromagnetic phase transition induced by temperature at zero external magnetic field is of second order. The magnetization continuously changes with temperature, with $M=0$ for $T>$ $T_{c}$ and $M>0$ for $T<T_{c}$, where $T_{c}$ is the material's specific Curie temperature. The vanishing of the magnetization with increasing temperature is characterized by the magnetization critical exponent $\beta$ governing the power-law behavior, $M \sim$ $\left(T_{c}-T\right)^{\beta}$. Near $T_{c}$, the divergence of the susceptibility $\chi$ is characterized by a susceptibility critical exponent $\gamma$ as $\chi^{-1} \sim\left(T-T_{c}\right)^{\gamma}$. Furthermore, exactly at $T_{c}$ the field-induced magnetization follows $M \sim H^{1 / \delta}$, with $\delta$ the field critical exponent. On the other hand, for fixed temperatures below $T_{c}$, a variation of the external magnetic field $H$ leads to a first-order transition when passing through $H=0$; this is a transition between two symmetric "up" and "down" magnetic states characterized by a magnetization jump $\Delta M$ which vanishes at $T_{c}$. Therefore, the magnetic phase transition is characterized by a temperature-dependent first-order line at $H=0$ which ends in a second-order critical point at $\left(T=T_{c}, H=0\right)$.

A very useful tool to study the second-order magnetic transition is the Arrott plot, developed more than 50 years ago [4]. It originates from an expansion of the system's free energy in terms of the magnetization and consists of plotting the isothermal magnetization data $M(H)$ as $H / M$ against $M^{2}$. In the Arrott plot, finite magnetic-field data correspond to straight lines which intercept the $H / M=0$ axis at positive values of $M_{0}^{2}(T)$ for $T<T_{c}$, with $M_{0}^{2}(T) \rightarrow 0$ when $T \rightarrow T_{c}$, whereas they intercept the $M^{2}=0$ axis at $\chi_{0}^{-1}(T)=(H / M)_{0}>0$ for $T>T_{c}$, which vanishes at $T_{c}$. Therefore, this simple method was first used to obtain values for the Curie temperature from isothermal magnetization curves through the extrapolation of $M^{2}(T)$ and $\chi^{-1}(T)$ to zero. This approach can be shown to be based on mean-field theory versions of the magnetic transition ( $\beta=1 / 2, \gamma=1$, and $\delta=3$ ) and was later generalized to the Arrott-Noakes plot [5], which considers different values for the critical exponents, i.e., different universality classes. The Arrott and Arrott-Noakes plots have then been widely used to investigate the second-order paramagnetic-ferromagnetic phase transition [6-14].

Another commonly used tool, also related to the Arrott plot, is the Banerjee criterion [15], which aims at experimentally distinguishing between first- and second-order temperaturedriven magnetic transitions through the sign of the slope of the $H / M$ against $M^{2}$ plot: a positive (negative) sign indicates a second-order (first-order) phase transition. The Banerjee criterion was originally inspired in the Bean-Rodbell theory for first-order magnetic transitions [16]. Within this theory a volume-magnetization coupling term is added to the molecular field model which then results in the possibility of a sign change for the fourth-order term of the magnetization expansion of the free energy. As stated by Banerjee, this method could provide a tool to distinguish first-order magnetic transitions from second-order ones by purely magnetic methods [15]. Since then, the Banerjee criterion has been widely used whenever indications of temperature-induced first-order transitions were observed, in particular for metamagnetic isothermal magnetic-field measurements [17-21]. As a drawback of the Banerjee criterion, it can be noticed that while the (negative) slope is expected to increase continuously with increasing temperature, as originally pointed out in Banerjee's work [15], this is not observed in most of the experimental examples which use the criterion to propose first-order transitions [21-24].

In the present work we use the Bean-Rodbell molecularfield model to critically discuss the use of the Arrott plot and 
Banerjee criterion to study magnetic phase transitions. We show that at zero magnetic field the $H-T$ phase diagram has a triple point joining three first-order critical lines, two of which end in second-order critical points at finite magnetic fields. We show then that a modified Arrott plot can be developed to be used around the second-order critical point, which is particularly useful to locate critical field and temperature values. In order to illustrate the presented picture we analyze experimental data for $\mathrm{La}_{2 / 3} \mathrm{Ca}_{1 / 3} \mathrm{MnO}_{3}$, an extensively studied material showing a first-order magnetic transition at low magnetic fields.

The paper is organized as follows. In Sec. II we introduce the model free-energy density and how the Arrott plot and the Banerjee criterion are usually derived and used to identify first-order magnetic transitions. In Sec. III we present the full phase diagram of the model, including the two symmetrical second-order critical points. In Sec. IV we show how to derive a modified Arrott plot close to the new second-order critical points and discuss its relation with the traditional Arrott plot. We next show in Sec. V magnetization measurements of $\mathrm{La}_{2 / 3} \mathrm{Ca}_{1 / 3} \mathrm{MnO}_{3}$ supporting the presence of second-order critical points at finite magnetic fields. Finally, Sec. VI summarizes our work.

\section{FREE-ENERGY MODEL}

The simplest free-energy model capturing second-order transition features is a Landau free-energy model obtained from an expansion in terms of the order parameter, where the coefficient accompanying the squared order parameter term can change its sign with varying temperature. In order to induce first-order characteristics, a strong variation of the coefficients of higher order terms in the free-energy expansion should be included. This has been originally achieved by introducing magnetization-volume coupling in magnetic models $[16,25]$ or within metamagnetic transition models for itinerant-electron systems [26].

In its simplest formulation, the molecular-field approximation captures the main features of mean-field paramagneticferromagnetic phase transitions, including the typical secondorder critical point and its critical exponents. Our starting point to study first-order magnetic transitions is a modified molecular-field approximation with a linear coupling between the magnetization $M$ and the specific volume $V$. This would correspond to systems where there is an interplay between the magnetic degrees of freedom and the crystal lattice leading to magnetostrictive transitions. This model was originally proposed and studied by Rodbell and co-workers [16,25]. Details of the model are included in Appendix A but we describe here the main features to derive a Landau-type free-energy density model.

Expanding the free-energy density around $M=0$, and truncating it to sixth order (see Appendix A), the resulting magnetic free-energy density reads

$$
\begin{aligned}
F_{M}= & \frac{N k_{B}}{2}\left(T-T_{c}\right)\left(\frac{M}{M_{S}}\right)^{2}+\frac{N k_{B} T}{12}\left(\frac{M}{M_{S}}\right)^{4} \\
& +\frac{N k_{B} T}{30}\left(\frac{M}{M_{S}}\right)^{6}-H M
\end{aligned}
$$

where we have also added the Zeeman term for the interaction with an external magnetic field $H$. In Eq. (1) $N$ is the number of magnetic ions per formula unit, $k_{B}$ is the Boltzmann constant, $M$ is the magnetization per formula unit, and $M_{S}$ is its saturation value. The temperature scale $T_{c}$ is proportional to the exchange interaction and corresponds, in principle, to the Curie temperature of the magnetic system.

On the other hand, there is a volume contribution to the free energy $F_{V}$. For simplicity, we consider a system with null specific heat at constant volume, $C_{V}=0$, constant isothermal compressibility $K$, and constant thermal expansion coefficient $\alpha$. Taking into account the coupling with an external pressure $P$ we obtain for the volume contribution to the free energy

$$
F_{V}=\frac{1}{2 K} \frac{\left(V-V_{0}\right)^{2}}{V_{0}}-\frac{T \alpha V}{K}+P V,
$$

where $V$ is the volume of the system per formula unit and $V_{0}$ corresponds to its zero-temperature reference value.

A natural way to couple magnetization and volume is to consider that magnetic exchange interactions can change with volume. This implies that the characteristic Curie temperature of the magnetic material can change, so one can write, up to first order in the volume,

$$
T_{c}=T_{0}\left[1+\eta \frac{\left(V-V_{0}\right)}{V_{0}}\right],
$$

where now $T_{0}$ is the Curie temperature of the pure magnetic system, i.e., without the volume-magnetization coupling. The factor $\eta$ measures the strength of this $M-V$ coupling and can be formally defined as

$$
\eta=\frac{V_{0}}{T_{0}} \frac{\partial T_{c}}{\partial V} .
$$

The linear variation of $T_{c}$ with $V$, Eq. (3), replaced in the freeenergy model Eq. (1) can be transformed into a free-energy coupling term of the form

$$
F_{M V}=-\eta \frac{N k_{B} T_{0}}{2} \frac{V-V_{0}}{V_{0}}\left(\frac{M}{M_{S}}\right)^{2} .
$$

Finally, using dimensionless quantities $m=M / M_{S}$, $v=\left(V-V_{0}\right) / V_{0}, \quad \tau=T / T_{0}, \quad h=2 H M_{S} /\left(N k_{B} T_{0}\right), \quad p=$ $2 P V_{0} /\left(N k_{B} T_{0}\right), K^{\prime}=K N k_{B} T_{0} /\left(2 V_{0}\right)$, and $\alpha^{\prime}=\alpha T_{0}$, a dimensionless free-energy density $f=2 F /\left(N k_{B} T_{0}\right)$ can then be written as

$$
f=f_{0}+f_{m}+f_{v}+f_{m v},
$$

where $f_{0}$ is independent both of $m$ and $v$, the pure magnetic contribution is

$$
f_{m}=(\tau-1) m^{2}+\frac{\tau}{6} m^{4}+\frac{\tau}{15} m^{6}-h m,
$$

and the volume contribution is

$$
f_{v}=\frac{1}{2 K^{\prime}} v^{2}-\frac{\alpha^{\prime}}{K^{\prime}} \tau v+p v .
$$

The coupling between magnetization and volume becomes

$$
f_{m v}=-\eta v m^{2} \text {. }
$$

We now seek for a model free-energy density described only in terms of the magnetization. Minimizing $f_{v}$ with respect to 
$v$ the equilibrium volume is given by

$$
v=v_{0}=\alpha^{\prime} \tau-p K^{\prime} .
$$

However, when the coupling term is included in $f$, the equilibrium volume now comes out from minimizing $f_{v}+f_{m v}$ with respect to $v$ and then becomes

$$
v=\alpha^{\prime} \tau-p K^{\prime}+\eta K^{\prime} m^{2}=v_{0}+\eta K^{\prime} m^{2} .
$$

This last result is next used to write the free-energy density $f$ as a function of the reduced magnetization $m$ only to obtain

$$
\begin{aligned}
f= & f_{0}-\frac{v_{0}^{2}}{2 K^{\prime}}+\left[(\tau-1)-\eta v_{0}\right] m^{2} \\
& +\left(\frac{\tau}{6}-\frac{1}{2} \eta^{2} K^{\prime}\right) m^{4}+\frac{\tau}{15} m^{6}-h m .
\end{aligned}
$$

Note here that $v_{0}$ also depends on the reduced temperature $\tau$. The free-energy density can finally be written in the form

$$
f=f_{0}^{\prime}+a\left(\tau-\tau_{1}\right) m^{2}+\frac{1}{6}\left(\tau-\tau_{2}\right) m^{4}+\frac{\tau}{15} m^{6}-h m,
$$

with

$$
f_{0}^{\prime}(\tau)=f_{0}-\frac{v_{0}^{2}(\tau)}{2 K^{\prime}} .
$$

The prefactor in the $m^{2}$ term is given by

$$
a=1-\eta \alpha^{\prime},
$$

and the two characteristic reduced temperatures are defined as

$$
\begin{gathered}
\tau_{1}=\frac{1-\eta p K^{\prime}}{1-\eta \alpha^{\prime}}, \\
\tau_{2}=3 \eta^{2} K^{\prime} .
\end{gathered}
$$

In terms of the original thermodynamic variables, the characteristic temperatures are

$$
\begin{aligned}
& T_{1}=T_{0} \frac{1-\eta P K}{1-\eta \alpha T_{0}}, \\
& T_{2}=\frac{3 \eta^{2} K N k_{B} T_{0}^{2}}{2 V_{0}} .
\end{aligned}
$$

When $\eta=0$ there is no coupling between $M$ and $V$, and these temperatures take the values $T_{1}=T_{0}=T_{c}$ and $T_{2}=0$, as expected.

When analyzing the free-energy density Eq. (13) in terms of the reduced magnetization, one realizes that the magnetic phase transition can be either first or second order, depending on the relative values of $\tau_{1}$ and $\tau_{2}$. For $h=0$ and at high temperatures, the system is in a paramagnetic state with $m=0$. If $\tau_{1} \geqslant \tau_{2}$, when the reduced temperature $\tau$ decreases, the $m^{2}$ term becomes negative when $\tau<\tau_{1}$ and there exists a temperature range with a positive $m^{4}$ term, i.e., $\tau_{2}<\tau<\tau_{1}$, thus corresponding to a second-order phase transition at $\tau=\tau_{1}$, similar to the standard zero-field temperature-driven magnetic transition. The expected behavior of the free-energy density for this case is presented in Fig. 1(a). When the temperature decreases from the paramagnetic region, if $\tau_{2}>\tau_{1}$ the $m^{4}$ term becomes negative before the $m^{2}$ term does. Therefore, the
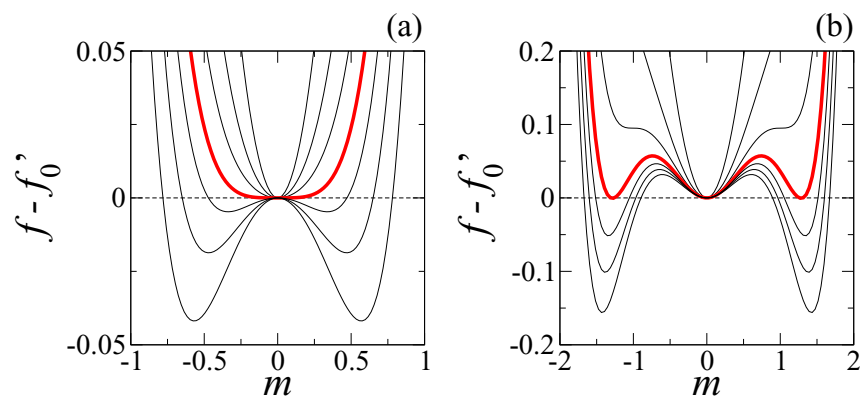

FIG. 1. (a) Second-order phase transition for $\tau_{1} \geqslant \tau_{2}$. Freeenergy density Eq. (13) with $a=0.8, \tau_{1}=3$, and $\tau_{2}=1$. Each curve corresponds, from top to bottom, to $\tau=4,3.4,3.15,3,2.9,2.8,2.7$ and the bold red line corresponds to $\tau=\tau_{c}=3$. (b) First-order phase transition for $\tau_{2}>\tau_{1}$. Free energy density, Eq. (13), with $a=0.8, \tau_{1}=1$, and $\tau_{2}=3$. Each curve corresponds, from top to bottom, to $\tau=2,1.5,1.35,1.293,1.27,1.25,1.23$ and the bold red line corresponds to $\tau=\tau^{*} \approx 1.293$.

free-energy density develops a second minimum at a value $m^{*}$ different from $m=0$ and when $f(m=0)=f\left(m=m^{*}\right)$ the system jumps from the high temperature equilibrium solution ( $m=0)$ to the new equilibrium solution with $m=m^{*}$, implying a discontinuous change on the magnetization. Figure 1(b) shows how the free-energy density changes with temperature in this case, which corresponds to a first-order phase transition at a reduced temperature $\tau^{*}$ such that $\tau_{1}<\tau^{*}<\tau_{2}$. This is the main result in Ref. [16] and it has been widely used to describe magnetic first-order transitions [7,27-31].

\section{PHASE DIAGRAM}

Though for $h=0$ the relative value of the characteristic temperatures $\tau_{1}$ and $\tau_{2}$ controls whether the magnetization increases discontinuously or not, with the transition acquiring a first- or second-order character, a complete and clearer picture emerges when considering finite values for the magnetic field $h$. For example, for $h \neq 0$ the isothermal free-energy density curves shown in Fig. 1(b) would tilt and a transition with a magnetization jump would appear for temperature values larger than $\tau^{*}$. This suggests the presence of a first-order transition line with $h \neq 0$. Therefore, in this section we present the full $h-\tau$ phase diagram for the free-energy density model Eq. (13). By minimizing the free-energy density we will compute the location of a triple point where three first-order lines meet, as well as the corresponding second-order critical points signaling the end of the first-order lines. We shall also characterize the behavior of the magnetization and the order parameters associated to the phase transitions.

For $h=0$ the high temperature phase is a paramagnetic state with $m=0$, while the low temperature phase is characterized by a finite magnetization $m(\tau)$ [32]. The values of the reduced temperature $\tau^{*}$ and the magnetization $m^{*}=m\left(\tau^{*}\right)$ at the $h=0$ temperature-driven transition correspond to the triple point and can be computed from

$$
\begin{gathered}
\left.\partial_{m} f(m, \tau, h=0)\right|_{m=m^{*}}=0, \\
f\left(m^{*}, \tau^{*}, h=0\right)=f\left(0, \tau^{*}, h=0\right) .
\end{gathered}
$$


From these conditions, the reduced transition temperature for $h=0$ is

$$
\tau^{*}=\frac{24 a \tau_{1}-5 \tau_{2}+4 d}{48 a-5},
$$

and the magnetization at the triple point is given by

$$
m^{* 2}=\frac{5}{3} \frac{12 a\left(2 \tau_{2}-\tau_{1}\right)+2 d-e}{24 a \tau_{1}-15 \tau_{2}+4 d},
$$

where we have defined the auxiliary quantities $d$ and $e$ through

$$
\begin{gathered}
d^{2}\left(\tau_{1}, \tau_{2}\right)=3 a\left[12 a \tau_{1}^{2}+5 \tau_{2}\left(\tau_{2}-\tau_{1}\right)\right] \\
e^{2}\left(\tau_{1}, \tau_{2}\right)=a\left[\left(\tau_{2}-\tau_{1}\right)\left[(24 a+5) \tau_{2}-\tau_{1}\right]+4\left(\tau_{1}-2 \tau_{2}\right) d\right] .
\end{gathered}
$$

When $h>0$ a negative linear term is added to the symmetric free-energy density of Fig. 1 and then the large temperature magnetization minimum is shifted to positive values; the system is therefore in a low-magnetic state $m_{1}$ at large temperatures. At small fields (below a field value $h_{c}$ to be defined below), when the temperature is decreased there is a first-order transition to a high-magnetic state $m_{2}$ at a field-dependent temperature $\tau^{\mathrm{FO}}(h)$. The temperature and field dependence of the high- and low-magnetic states can be obtained from the equation of state (defined by $\partial f / \partial m=0$ )

$$
2 a\left(\tau-\tau_{1}\right) m+\frac{2}{3}\left(\tau-\tau_{2}\right) m^{3}+\frac{6}{15} \tau m^{5}-h=0,
$$

supplemented by the fact that the equilibrium magnetization is the solution of the equation of state with minimum free energy. The magnetization jump at the transition from lowto high-magnetic states is $\Delta m^{\mathrm{FO}}=m_{2}-m_{1}$ and decreases with increasing field. Figure 2 shows the evolution of the magnetization $m$ showing the characteristic jump between low- and high-magnetic states, $m_{1}$ and $m_{2}$, respectively, for finite $\tau$ and $h$ values. This first-order phase transition occurs at a field-dependent temperature $\tau^{\mathrm{FO}}(h)$ given by the condition

$$
f\left(m_{1}\left(\tau^{\mathrm{FO}}, h\right), \tau^{\mathrm{FO}}, h\right)=f\left(m_{2}\left(\tau^{\mathrm{FO}}, h\right), \tau^{\mathrm{FO}}, h\right) .
$$

(a)

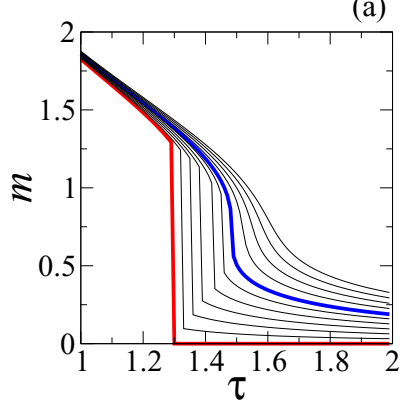

(b)

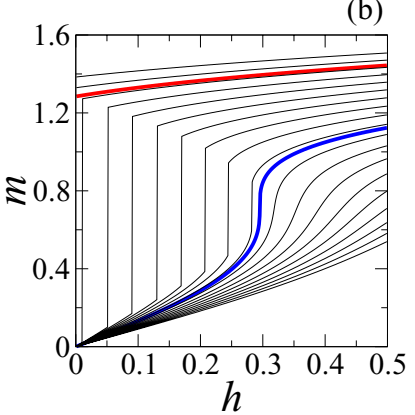

FIG. 2. Evolution of the magnetization $m$ corresponding to the free-energy density Eq. (13) for $a=0.8$. (a) Magnetizationtemperature curves, $m(\tau)$, for different field values $h=$ $0,0.05,0.1, \ldots, 0.5$. The thick red and blue lines correspond to $h=0$ and $h_{c}=0.295$, respectively. (b) Magnetization-field curves, $m(h)$, for different temperature values $\tau=1.25,1.275,1.3, \ldots, 1.7$. The thick red and blue lines correspond to $\tau^{*} \approx 1.293$ and $\tau_{c} \approx 1.484$, respectively.

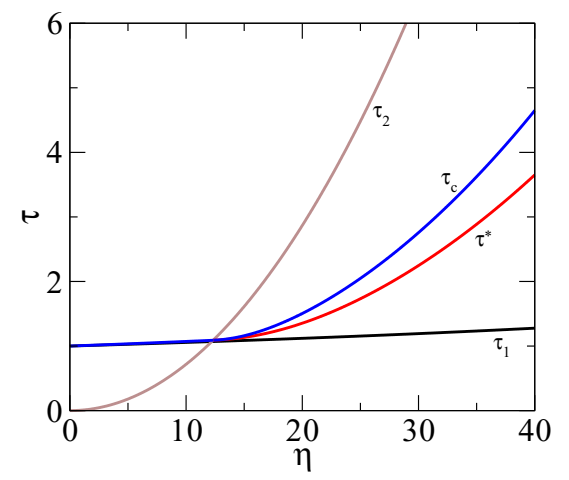

FIG. 3. Dependence of the dimensionless characteristic temperatures $\tau_{1}, \tau_{2}, \tau_{c}$, and $\tau^{*}$ on the coupling parameter $\eta$.

The jump $\Delta m^{\mathrm{FO}}$ vanishes at a given critical field value. Above this value, the two magnetic states can no longer be distinguished. This defines the second-order critical point of the magnetic transition characterized by $h_{c}, \tau_{c}$, and $m_{c}$. In terms of the free energy this corresponds to the absence of an inflexion point. From this criterium one obtains

$$
\begin{gathered}
\tau_{c}=\frac{2 a \tau_{1}-\tau_{2}+2 d^{\prime}}{4 a-1}, \\
h_{c}=16 a\left[\frac{a\left(-\tau_{1}+2 \tau_{2}\right)-d^{\prime}}{2 a \tau_{1}-\tau_{2}+2 d^{\prime}}\right]^{3 / 2} \\
\times \frac{\tau_{1} \tau_{2}-2 a\left(\tau_{1}^{2}-2 \tau_{1} \tau_{2}+2 \tau_{2}^{2}\right)-2 \tau_{1} d^{\prime}-\tau_{2}\left(\tau_{2}-4 d^{\prime}\right)}{15(4 a-1)\left(a\left(\tau_{1}-2 \tau_{2}\right)+d^{\prime}\right)}, \\
m_{c}=\sqrt{\frac{a\left(-\tau_{1}+2 \tau_{2}\right)-d^{\prime}}{2 a \tau_{1}-\tau_{2}+2 d^{\prime}}}
\end{gathered}
$$

where we have now defined

$$
d^{\prime 2}\left(\tau_{1}, \tau_{2}\right)=a\left(a \tau_{1}^{2}+\tau_{2}\left(\tau_{2}-\tau_{1}\right)\right)
$$

The relative values of the characteristic temperature scales are controlled by the coupling parameter $\eta$, defined in Eq. (4). The dependence of the characteristic temperatures $\tau_{1}, \tau_{2}$, $\tau^{*}$, and $\tau_{c}$ on the coupling parameter $\eta$ are presented in Fig. 3. These values were computed using dimensionless values at ambient pressure $K^{\prime}=2.39 \times 10^{-3}, p=0.31$, and $\alpha^{\prime}=6 \times 10^{-3}$, estimated from $\mathrm{La}_{2 / 3} \mathrm{Ca}_{1 / 3} \mathrm{MnO}_{3}$ parameters: $T_{0}=T_{c}^{\exp } / b$, with $T_{c}^{\exp }=260 \mathrm{~K}$ (Refs. [33,34]) and $b=$ 1.3 (Ref. [25]), $\alpha=3 \times 10^{-5} \mathrm{~K}^{-1}$ (Ref. [35]), $K=7.4 \times$ $10^{-11} \mathrm{~Pa}^{-1}$ (Ref. [36]), and $V_{0}=57.75 \AA^{3}$ (Ref. [33]). When $\tau_{1} \geqslant \tau_{2}$ the transition is of second order and $\tau_{c}=\tau^{*}=\tau_{1}$. Instead, when $\tau_{2}>\tau_{1}$ the transition is of first order, occurring at a field-dependent temperature $\tau^{\mathrm{FO}}(h)$ between $\tau^{*}$ and $\tau_{c}$, and with both $\tau^{*}$ and $\tau_{c}$ having values bounded by $\tau_{1}$ and $\tau_{2}$, i.e., $\tau_{2}>\tau_{c}>\tau^{*}>\tau_{1}$. It should be noticed that the fact that $\tau_{2}>\tau_{c}>\tau^{*}$ was obtained in Ref. [26] in the context of metamagnetic transitions in itinerant-electron systems, but without exploiting the second-order character of the transition at $\tau_{c}$. 


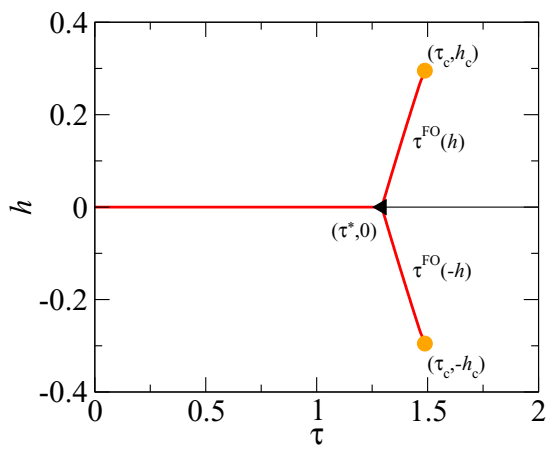

FIG. 4. $h-\tau$ phase diagram for the free-energy density model Eq. (13). Red lines are first-order critical lines. The black triangle corresponds to the triple point at $\left(\tau^{*}, h=0\right)$ while the orange circles at $\left(\tau_{c}, \pm h_{c}\right)$ are second-order critical points.

Figure 4 shows the $h-\tau$ phase diagram corresponding to the free-energy density Eq. (13) with $a=0.8$. The triangle indicates the triple point at $\left(\tau^{*}, h=0\right)$ where three first-order lines converge (red lines). The first-order line with $\tau<\tau^{*}$ and $h=0$ separates symmetric states with high magnetization, $\pm m_{2}$. The first-order lines $\tau^{\mathrm{FO}}(h)$ were obtained numerically from Eq. (27) and separate low- and high-magnetic states $m_{1,2}$. The orange circles indicate the end of the first-order lines in two symmetric second-order critical points $\left(\tau_{c}, \pm h_{c}\right)$.

\section{ARROTT PLOT}

We will show in the following how the Arrott plot is constructed and how it can be modified to study second-order critical points at finite magnetic fields.

The traditional Arrott plot is constructed by plotting $h / m$ against $m^{2}$. For a generic second-order phase transition described by the free-energy density

$$
f=f_{0}+\frac{A}{2}\left(\tau-\tau_{c}\right) m^{2}+\frac{B}{4} m^{4}-h m,
$$

where $A$ and $B$ are positive constants and $\tau_{c}$ is the critical reduced temperature. The equation of state, given by $\partial f / \partial m=$ 0 , is

$$
A\left(\tau-\tau_{c}\right) m+B m^{3}-h=0 .
$$

Equivalently

$$
\frac{h}{m}=A\left(\tau-\tau_{c}\right)+B m^{2}=g\left(m^{2}\right),
$$

with $g(x)=A\left(\tau-\tau_{c}\right)+B x$. Therefore, when plotting $h / m$ as a function of $m^{2}$ one obtains a simple linear behavior where the slope is associated to the fourth-order term in the freeenergy density. The extrapolation of these lines to $m^{2}=0$ is temperature dependent, and such that its value is positive for $\tau>\tau_{c}$ and negative otherwise, thus allowing a simple way to locate the value of the critical temperature. This simple picture can be slightly modified if the positive parameters $A$ and $B$ depend on temperature, as expected under experimental conditions. Moreover, this picture can be strongly affected when other terms in the free-energy density are considered, such as those coupling the two degrees of freedom $M$ and $V$ as discussed in the previous section. (a)
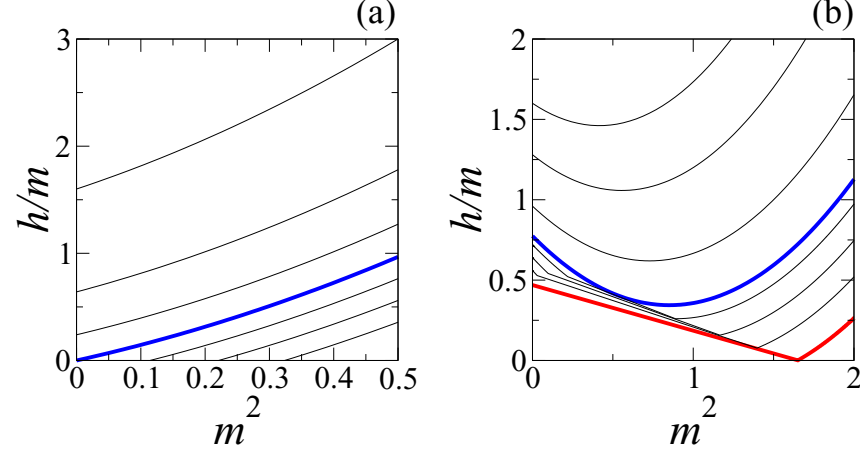

FIG. 5. Arrott plots corresponding to Eq. (35) for the free-energy densities of Fig. 1. (a) Second-order phase transition for $\tau_{1} \geqslant \tau_{2}$. The curves correspond to $a=0.8, \tau_{1}=3$, and $\tau_{2}=1$. From top to bottom, they were computed using $\tau=4,3.4,3.15,3,2.9,2.8,2.7$ and the blue bold line corresponds to $\tau_{c}=3$. (b) First-order phase transition for $\tau_{2}>\tau_{1}$. The curves are for $a=0.8, \tau_{1}=1$, and $\tau_{2}=3$. Each curve corresponds, from top to bottom, to $\tau=$ $2,1.8,1.6,1.484,1.45,1.4,1.35,1.293$ and the blue and red bold lines correspond to $\tau_{c} \approx 1.484$ and $\tau^{*} \approx 1.293$, respectively.

In such a case, going back to Eq. (13) for the free-energy density and its corresponding equation of state, Eq. (26), one gets

$$
\frac{h}{m}=2 a\left(\tau-\tau_{1}\right)+\frac{2}{3}\left(\tau-\tau_{2}\right) m^{2}+\frac{6}{15} \tau m^{4} .
$$

Using this expression, Arrott plots $h / m$ against $m^{2}$ are constructed, and shown in Fig. 5. The Arrott plot corresponding to the second-order phase transition for $\tau_{1} \geqslant \tau_{2}$, Fig. 5(a), always shows a positive slope. In addition, the positive curvature observed is due to the term proportional to $m^{6}$ in the free-energy density. If this term was absent, only straight lines would be observed. On the other hand, the Arrott plot for the case $\tau_{2}>\tau_{1}$, Fig. 5(b), shows curves with rather different behavior depending on the temperature. For temperatures larger than $\tau_{2}$ the right-hand side of Eq. (35) is positive and all curves have a positive slope [not shown in Fig. 5(b)]. Instead, for temperatures below $\tau_{2}$ a negative slope is expected. In the range $\tau_{c}<\tau<\tau_{2}$ isothermal continuous curves present a minimum value but when $\tau<\tau_{c}$ a discontinuous jump in the Arrott plot is observed. This discontinuity reflects the equilibrium magnetization jump through the first-order transition, as shown in Fig. 6 where the relation between the magnetization-field curves and the Arrott plot is highlighted for $\tau_{c}$ and $\tau=1.4=0.943 \tau_{c}$. Since only equilibrium solutions of the equation of state must be used, the isothermal curve for $\tau=0.943 \tau_{c}$ in the Arrott plot is necessarily discontinuous. Finally, for temperatures below $\tau^{*}$ only the large $m^{2}$ part of the Arrott plot would appear in Fig. 5(b) (not shown) for $m^{2}>\left(m^{*}\right)^{2}$, since in this case the field-induced jump is between $\pm m_{2}$ magnetization states. Therefore, in the case $\tau_{2}>\tau_{1}$ there is a temperature range where a negative slope can be expected in the Arrott plot, as noticed by Banerjee [15]. 
(a)
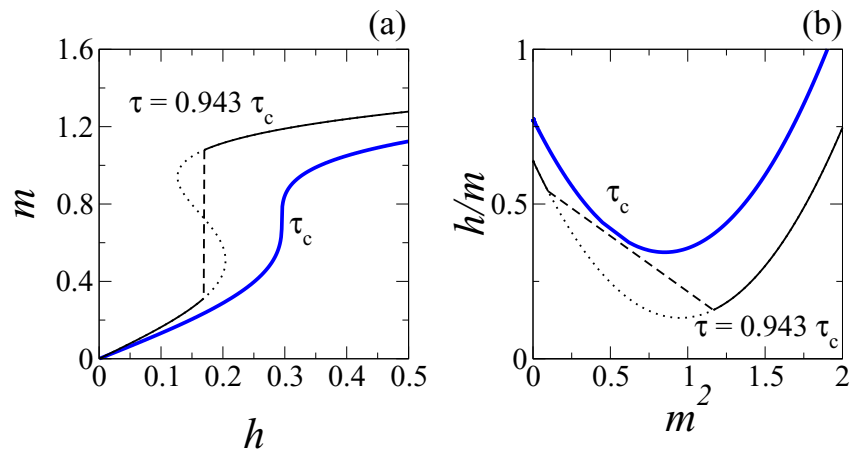

FIG. 6. (a) Magnetization-field curve and (b) Arrott plot for $\tau_{c}=$ 1.484 and a temperature $\tau=0.943 \tau_{c}$. The magnetization jump for $\tau=0.943 \tau_{c}$ in (a) leads to the discontinuity in the Arrott plot in (b) (dashed lines). Dotted lines represent the nonequilibrium solutions to the equation of state.

\section{A. Banerjee criterion}

The relationship between the molecular-field model of Bean and Rodbell and the mean-field Landau model for secondorder magnetic transitions led Banerjee to point out that the negative slope in Arrott plots can be a good criterion to identify first-order magnetic transitions [15]. This simple and powerful idea has been used ever since as a proof of the first-order character of a magnetic transition.

Although the negative sign of the slope in an Arrott plot can be considered as a clear signature of an underlying first-order transition, Banerjee also made a second important observation: the theory predicts the value of the (negative) slope to increase with increasing temperature [15]. In fact, it follows from Eq. (35) that the slope of the Arrott, when $m^{2} \rightarrow 0$, is

$$
s=\frac{\partial(h / m)}{\partial\left(m^{2}\right)}=\frac{2}{3}\left(\tau-\tau_{2}\right),
$$

and so it changes sign at $\tau=\tau_{2}>\tau_{c}>\tau^{*}$. This observation contrasted with the experimental data for MnAs presented in Ref. [15], as noticed by the author. More importantly, most of the subsequent works using the Banerjee criterion to identify first-order transitions show that the (negative) slope of the Arrott plot, $S=\partial(H / M) / \partial\left(M^{2}\right)$, decreases when increasing the temperature, as shown in Fig. 7 for some selected examples in the literature [24,31,34]. These results clearly depart from the mean-field approximation, Eq. (36), shown in Fig. 7(d).

\section{B. Arrott plot close to a second-order critical point}

It is instructive to analyze the physics around the secondorder critical points $\left(\tau_{c}, \pm h_{c}\right)$. The first-order line ending on the second-order critical point separates two states with finite magnetization, $m_{1}$ and $m_{2}$, and then the free-energy density is no longer symmetric with respect to the magnetization. We then seek for a symmetric expansion of the free energy close to the critical point, thus only containing even powers of the order parameter. Details are given in Appendix B and we describe here only the main physical picture. We can write the magnetization around the critical point as

$$
m=m_{c}+\phi_{0}+\Delta \phi,
$$

(a)

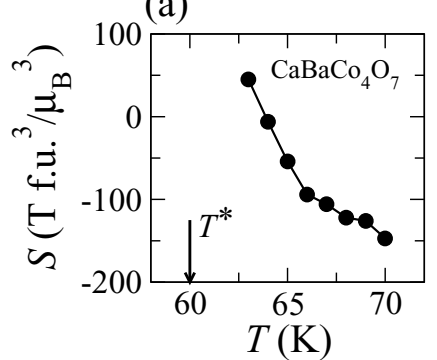

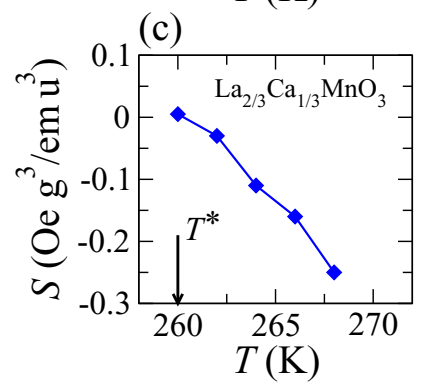

(b)

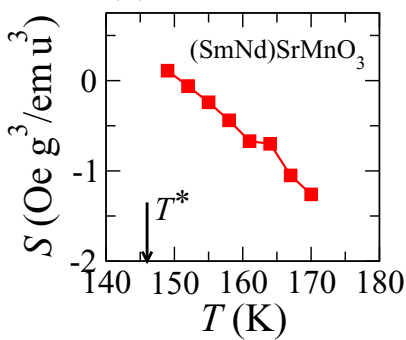

(d)

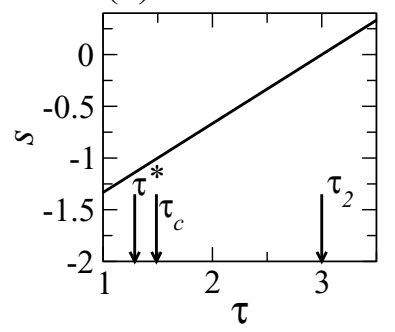

FIG. 7. Temperature dependence of the slopes of $\mathrm{Ar}$ rott plots corresponding to (a) $\mathrm{CaBaCo}_{4} \mathrm{O}_{7}$ (Ref. [24]), (b) $\left(\mathrm{Sm}_{0.7} \mathrm{Nd}_{0.3}\right)_{0.52} \mathrm{Sr}_{0.48} \mathrm{MnO}_{3}$ (Ref. [31]), and (c) $\mathrm{La}_{2 / 3} \mathrm{Ca}_{1 / 3} \mathrm{MnO}_{3}$ (Ref. [34]). (d) Expected temperature dependence of the Arrott plot slope from the free-energy model, Eq. (36). The zero-field transition temperatures $T^{*}$ are indicated in (a)-(c), while the three characteristic temperatures of the mean-field model are indicated in (d).

where $\Delta \phi$ is the new order parameter and $\phi_{0}$ guarantees a symmetric solution. Up to first order in $\Delta \tau=\tau-\tau_{c}$ (see Appendix B) we have

$$
\phi_{0}=\frac{1+2 m_{c}^{2}}{6 \tau_{c} m_{c}} \Delta \tau=\widetilde{\phi}_{0} \Delta \tau .
$$

The free-energy density can now be expanded around the critical point to obtain

$$
\widetilde{f}=f_{0}^{\prime \prime}+\frac{1}{2} \widetilde{r}\left(\tau-\tau_{c}\right) \Delta \phi^{2}+u \Delta \phi^{4},
$$

where $f_{0}^{\prime \prime}=f\left(\phi_{0}\right)$ and

$$
\begin{gathered}
\tilde{r}=2\left(a+m_{c}^{2}+m_{c}^{4}\right), \\
u=\tau_{c} m_{c}^{2} .
\end{gathered}
$$

This free-energy density has, as expected, the standard form for a second-order phase transition with an order parameter $\Delta \phi$ such that the stable solutions are

$$
\Delta \phi= \begin{cases}0 & \text { for } \tau>\tau_{c}, \\ \Delta \phi_{1,2} & \text { for } \tau<\tau_{c},\end{cases}
$$

with

$$
\Delta \phi_{1,2}= \pm \sqrt{-\frac{\widetilde{r}\left(\tau-\tau_{c}\right)}{4 u}} .
$$

The two low-temperature symmetric solutions $\Delta \phi_{1,2}$ correspond to the nonsymmetric low- and high-magnetic states $m_{1,2}=m_{c}+\phi_{0} \pm \Delta \phi_{0}$ close to the critical point.

The conjugated field $\mu$ associated to the order parameter $\Delta \phi$ can be shown to be field and temperature dependent 
(Appendix B) and, up to first order in $\Delta h=h-h_{c}$ and $\Delta \tau$, it is given by

$$
\mu=\Delta h-\tilde{\mu} \Delta \tau
$$

with

$$
\tilde{\mu}=2 m_{c}\left(a+\frac{1}{3} m_{c}^{2}+\frac{1}{5} m_{c}^{4}\right) .
$$

Therefore, adding a term $-\mu \Delta \phi$ to the free-energy Eq. (39) favors the $+\Delta \phi$ solution as usual. This represents a standard second-order phase transition, and then the Arrott plot in terms of the variables $(\mu, \Delta \phi)$ is well behaved when plotting $\mu / \Delta \phi$ against $\Delta \phi^{2}$ since

$$
\frac{\mu}{\Delta \phi}=\widetilde{r}\left(\tau-\tau_{c}\right)+4 u \Delta \phi^{2} .
$$

The next step is to use this well behaved Arrott plot to construct the modified Arrott plot close to the second-order critical point using the original variables $(h, m)$. Recalling that $\mu=\Delta h-\tilde{\mu} \Delta \tau$ and $\Delta \phi=m-m_{c}-\phi_{0}$ the Arrott relation Eq. (46) becomes

$$
\begin{aligned}
& \frac{h-h_{c}-\tilde{\mu}\left(\tau-\tau_{c}\right)}{m-m_{c}-\widetilde{\phi}_{0}\left(\tau-\tau_{c}\right)} \\
& \quad=\widetilde{r}\left(\tau-\tau_{c}\right)+4 u\left(m-m_{c}-\widetilde{\phi}\left(\tau-\tau_{c}\right)\right)^{2},
\end{aligned}
$$

where $\tilde{\mu}, \widetilde{\phi}_{0}, \widetilde{r}$, and $u$ are defined above in terms of $a, m_{c}$, and $\tau_{c}$. This modified Arrott relation contains field and temperature corrections to the external field and the magnetization, taking into account the asymmetry of the free-energy density with respect to the magnetization close to the second-order critical point at $\left(\tau_{c}, \pm h_{c}\right)$.

Finally, it can be noticed that close to the secondorder critical point one can write $h-h_{c}-\tilde{\mu}\left(\tau-\tau_{c}\right)=$ $h-h^{\mathrm{FO}}(\tau)$, with $h^{\mathrm{FO}}(\tau)=h_{c}+\tilde{\mu}\left(\tau-\tau_{c}\right)$ the temperature dependent critical field where the first-order transition takes place, i.e., $h^{\mathrm{FO}}(\tau)$ is the inverse function of $\tau^{\mathrm{FO}}(h)$. Analogously, $m^{\mathrm{FO}}(\tau)=m_{c}+\widetilde{\phi}_{0}\left(\tau-\tau_{c}\right)=\left(m_{1}+m_{2}\right) / 2$ is the average magnetization which close to $\tau_{c}$ tends to the critical magnetization linearly with $\tau$. In terms of $h^{\mathrm{FO}}(\tau)$ and $m^{\mathrm{FO}}(\tau)$ the modified Arrott relation can then be recast as

$$
\frac{h-h^{\mathrm{FO}}(\tau)}{m-m^{\mathrm{FO}}(\tau)}=\widetilde{r}\left(\tau-\tau_{c}\right)+4 u\left(m-m^{\mathrm{FO}}(\tau)\right)^{2} .
$$

This modified form of the Arrott plot serves to analyze secondorder magnetic transitions at finite magnetic field, as we shall show in the next section.

\section{EXPERIMENTAL RESULTS}

As an example, we present here experimental results analyzed on the basis of the theory discussed in the present manuscript. The studied sample was $\mathrm{La}_{2 / 3} \mathrm{Ca}_{1 / 3} \mathrm{MnO}_{3}$, for which Arrott plots close to the zero-field magnetic transition have been already reported [34,37,38]. The sample was synthesized by solid state reaction at $T=1300^{\circ} \mathrm{C}$ as described elsewhere [39]. Magnetization data were collected using a commercial vibrating sample magnetometer with a magnetic field $H$ up to $10 \mathrm{kOe}$, in the temperature range from 200 to $300 \mathrm{~K}$.

Figure 8(a) shows the $M(T)$ curves for different field values $H$ as indicated, while the $M(H)$ curves for temperature

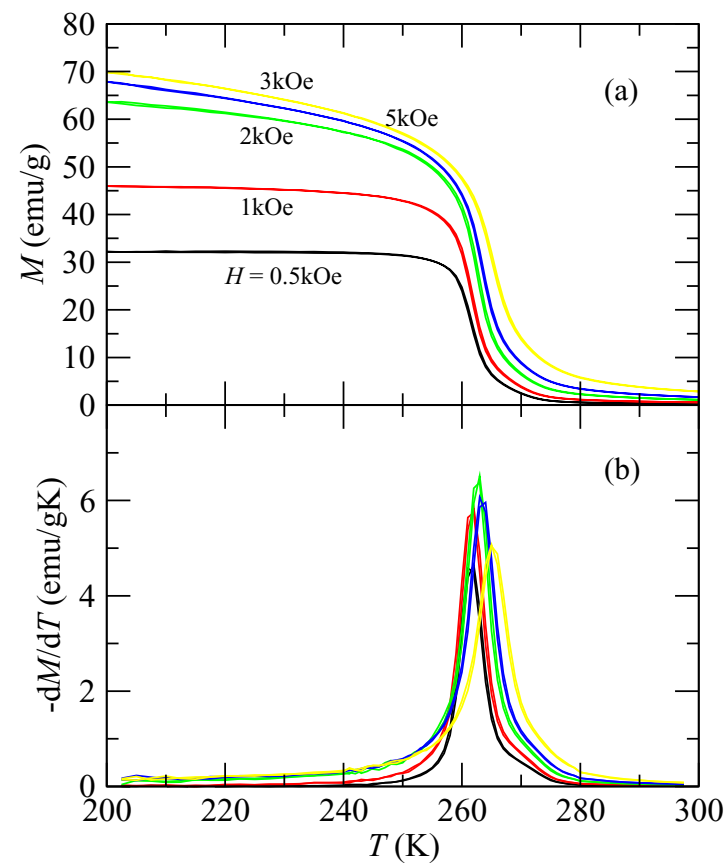

FIG. 8. Magnetization data for $\mathrm{La}_{2 / 3} \mathrm{Ca}_{1 / 3} \mathrm{MnO}_{3}$. (a) $M(T)$ curves at different magnetic-field values $H$ as indicated. (b) Numerical derivative $d M / d T$ of the data presented in (a).

values in the range $262 \mathrm{~K}<T<267 \mathrm{~K}$ are presented in Fig. 9(a). As can be observed in Fig. 2(a) the maximum derivatives of $m(\tau)$ for different values of $h$ give the transition temperature $\tau^{\mathrm{FO}}(h)$. Analogously, the maximum derivatives

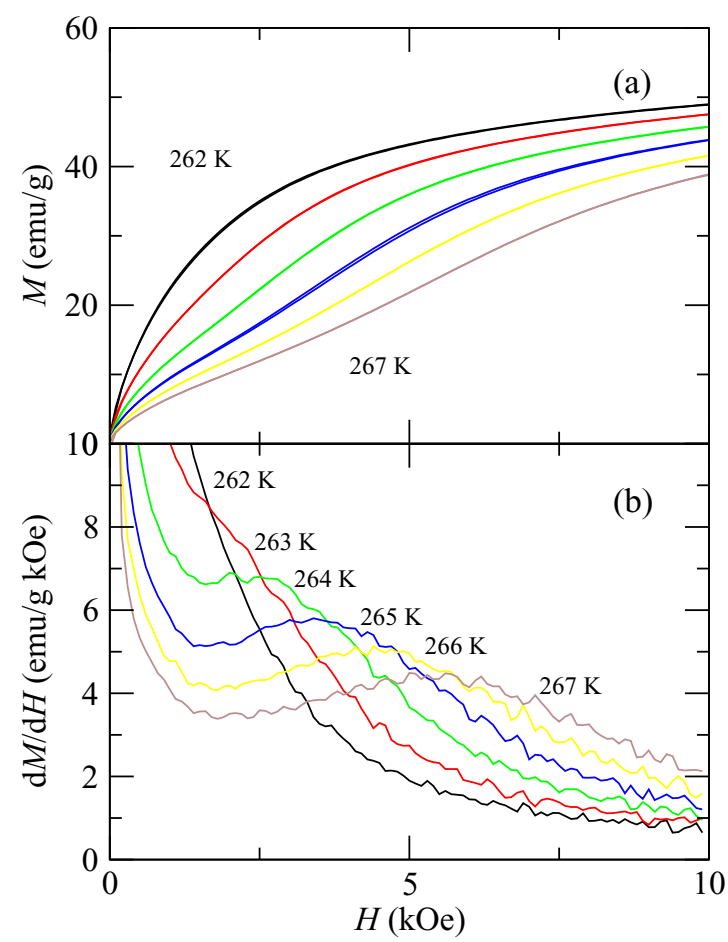

FIG. 9. Magnetization data for $\mathrm{La}_{2 / 3} \mathrm{Ca}_{1 / 3} \mathrm{MnO}_{3}$. (a) $M(H)$ curves at different temperatures $T$ as indicated. (b) Numerical derivative $d M / d H$ of the data presented in (a). 


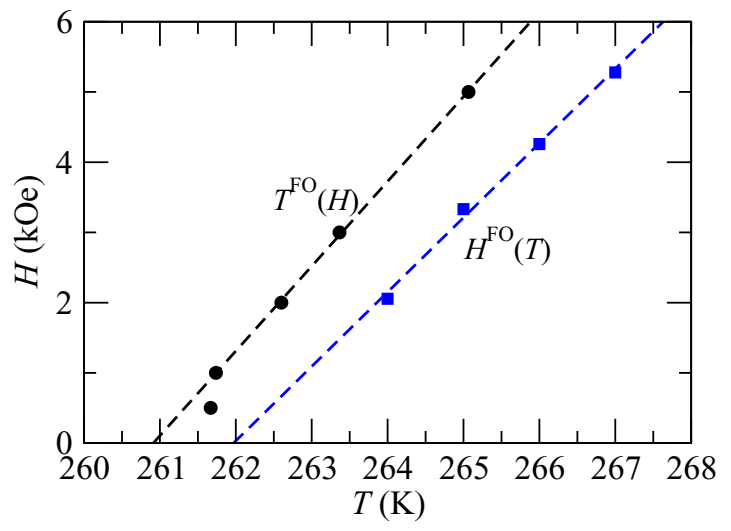

FIG. 10. Characteristic temperature $T^{\mathrm{FO}}(H)$ obtained from the maxima of $d M / d T$ in Fig. 8 (black circles) and characteristic field $H^{\mathrm{FO}}(T)$ obtained from the maxima of $d M / d H$ in Fig. 9 (blue squares). Dashed lines correspond to linear fits. For $T^{\mathrm{FO}}$ the first point was not included in the fit.

of $m(h)$ give $h^{\mathrm{FO}}(\tau)$ as observed in Fig. 2(b), with both $\tau^{\mathrm{FO}}(h)$ and $h^{\mathrm{FO}}(\tau)$ corresponding to the same first-order transition line. Figures $8(\mathrm{~b})$ and $9(\mathrm{~b})$ show the numerical derivatives of $M(T)$ and $M(H)$ for the experimental data in Figs. 8(a) and 9(a), respectively. The obtained characteristic values for $T^{\mathrm{FO}}(H)$ and $H^{\mathrm{FO}}(T)$ are shown in Fig. 10. These data indicate a possible first-order transition line in the $H-T$ phase space, as discussed previously. Dashed lines in Fig. 10 correspond to a linear fit of the two data sets. Notice that a linear extrapolation to $H=0$ from these data gives estimates for the zero-field transition temperatures $T^{*}=261 \mathrm{~K}$ and $T^{*}=262 \mathrm{~K}$ from $T^{\mathrm{FO}}(H)$ and from $H^{\mathrm{FO}}(T)$, respectively, consistent with previous results $[33,34]$.

The bare Arrott plot, $H / M$ against $M^{2}$, is constructed from the data in Fig. 9 and shown in Fig. 11. It can clearly be observed that the Arrott plot close to $T^{*}$ does not behave as a straight line as expected for standard second-order phase transitions. Furthermore, a tendency to develop a negative slope can be appreciated with a negative slope for $T=267 \mathrm{~K}$. This kind of behavior has been typically ascribed to first-order ferromagnetic transitions. Note however that the negative slope is experimentally observed to decrease with increasing

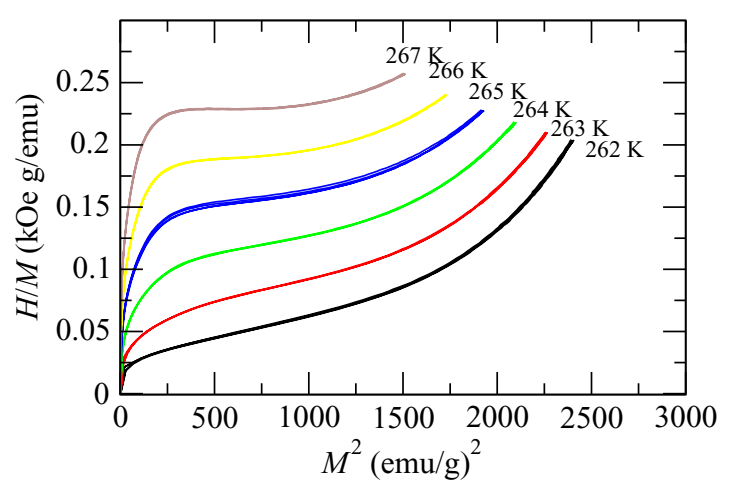

FIG. 11. Bare Arrott plot, $H / M$ against $M^{2}$, from the $M(H)$ data presented in Fig. 9 for $\mathrm{La}_{2 / 3} \mathrm{Ca}_{1 / 3} \mathrm{MnO}_{3}$.

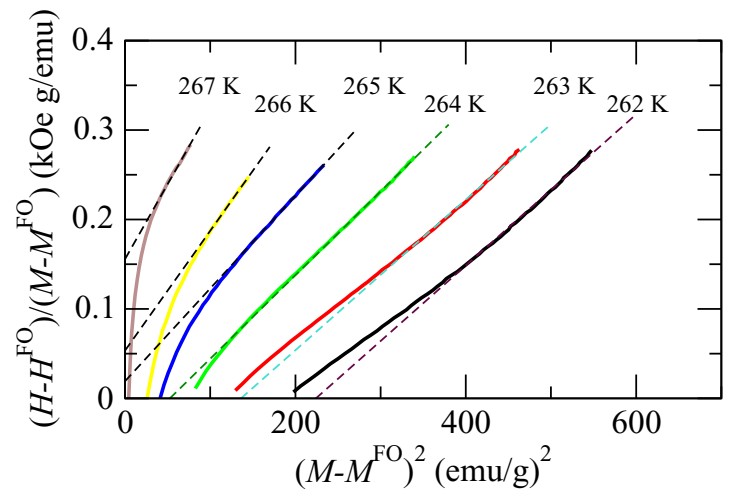

FIG. 12. Modified Arrott plot for $\mathrm{La}_{2 / 3} \mathrm{Ca}_{1 / 3} \mathrm{MnO}_{3}$ obtained from the $M(H)$ data in Fig. 9 and the temperature-dependent values for $H^{\mathrm{FO}}$ and $M^{\mathrm{FO}}$ shown in Table I. The linear fits for large $M-M^{\mathrm{FO}}$ are shown.

temperature (see also Refs. [34,38]), contrary to Eq. (36) as discussed in Sec. IV A.

We seek now for evidence of the presence of a second-order critical point at a finite $H$ value, i.e., $\left(T_{c}, H_{c}\right)$. Following the ideas presented in Sec. IV B, in Fig. 12 an alternative Arrott plot close to the second-order critical point is presented. Following Eq. (48), the modified Arrott plot shown in Fig. 12 is constructed by plotting $\left(H-H^{\mathrm{FO}}\right) /\left(M-M^{\mathrm{FO}}\right)$ against $\left(M-M^{\mathrm{FO}}\right)^{2}$. In order to produce this modified Arrott plot, the values of $M^{\mathrm{FO}}(T)=M_{c}-\bar{\phi}_{0} \Delta T$ and $H^{\mathrm{FO}}(T)=H_{c}-\bar{\mu} \Delta T$, with $\bar{\phi}_{0}=\widetilde{\phi}_{0} M_{S} / T_{0}$ and $\bar{\mu}=\tilde{\mu} N k_{B} / T_{0}$, need to be known. In principle, $H^{\mathrm{FO}}(T)$ represents the linear approximation to the first-order transition line close to the second-order critical point, while $M^{\mathrm{FO}}(T)$ corresponds to the average magnetization state close to $T_{c}$. One could argue that the values for $T^{\mathrm{FO}}$ or $H^{\mathrm{FO}}$, obtained from Fig. 10, and their corresponding $M^{\mathrm{FO}}$, could be good approximations to these quantities. However, we have found that the Arrott plot using these quantities presents large deviations from the expected simple second-order critical point behavior. In order to obtain the Arrott plot of Fig. 12 we used, instead, the values of $H^{\mathrm{FO}}(T)$ and $M^{\mathrm{FO}}(T)$ presented in Table I.

Next, we perform linear fits of the large $\left(M-M^{\mathrm{FO}}\right)^{2}$ data in the modified Arrott plot, as shown with dashed lines in Fig. 12. We then obtain $\left(H-H^{\mathrm{FO}}\right) /\left(M-M^{\mathrm{FO}}\right)_{0}$ and $\left(M-M^{\mathrm{FO}}\right)_{0}^{2}$ as the extrapolations of the linear fits to the $\left(M-M^{\mathrm{FO}}\right)^{2}=0$ and $\left(H-H^{\mathrm{FO}}\right) /\left(M-M^{\mathrm{FO}}\right)=0$ axes, respectively, which are shown in Fig. 13 as a function of temperature. As can

TABLE I. Temperature-dependent characteristic field $H^{\mathrm{FO}}$ and magnetization $M^{\mathrm{FO}}$ used to construct the modified Arrott plot shown in Fig. 12.

\begin{tabular}{lcc}
\hline \hline Temperature $(\mathrm{K})$ & $H^{\mathrm{FO}}(\mathrm{kOe})$ & $M^{\mathrm{FO}}(\mathrm{emu} / \mathrm{g})$ \\
\hline 262 & 3.5 & 25.5 \\
263 & 4 & 26 \\
264 & 5 & 27.3 \\
265 & 6 & 28.5 \\
266 & 7 & 29.5 \\
267 & 7.5 & 30 \\
\hline \hline
\end{tabular}




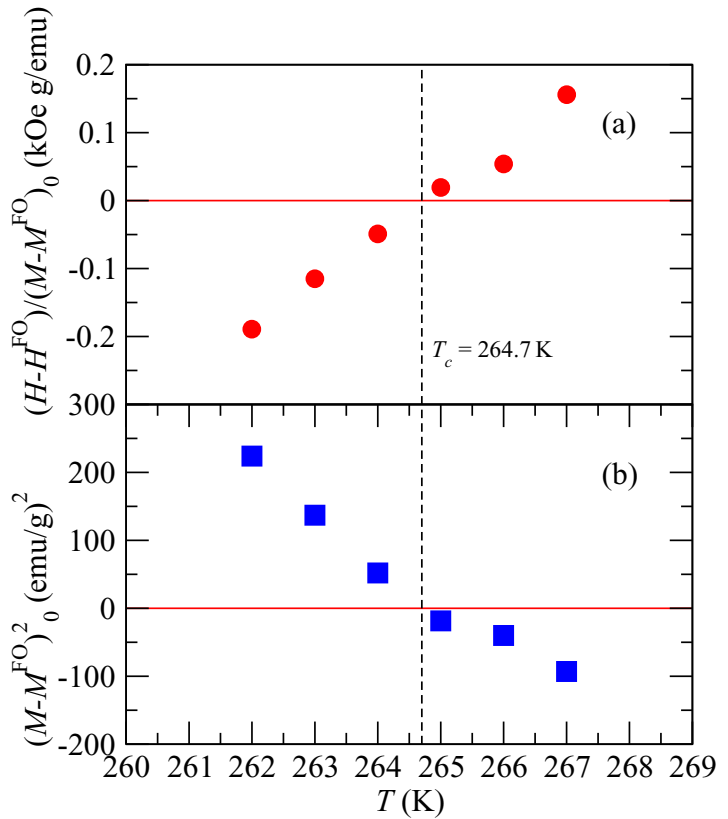

FIG. 13. Temperature dependence of (a) effective inverse susceptibility $\left(H-H^{\mathrm{FO}}\right) /\left(M-M^{\mathrm{FO}}\right)_{0}$ and (b) effective squared magnetization $\left(M-M^{\mathrm{FO}}\right)_{0}^{2}$. The change in sign of both quantities is compatible with a second-order critical temperature $T_{c}=264.7 \mathrm{~K}$, obtained from linear interpolation between the points with $T=264 \mathrm{~K}$ and $265 \mathrm{~K}$.

be observed, both quantities change sign, thus indicating the location of the critical temperature at $T_{c}=264.7 \mathrm{~K}$, as expected for second-order phase transitions. Therefore, this is compatible with the phase diagram presented in Fig. 4 and the existence of a second-order critical point, and hence support the use of the values for $H^{\mathrm{FO}}$ and $M^{\mathrm{FO}}$ given in Table I. What we show in this example is that there exists a set of $\left\{H^{\mathrm{FO}}, M^{\mathrm{FO}}\right\}$ values for which a modified Arrott plot can be constructed where a second-order critical behavior is recovered and may be analyzed within the mean-field theory for standard second-order phase transitions.

\section{SUMMARY}

In summary, we have presented here a deeper discussion on the phase diagram of magnetic phase transitions and the relevance of Arrott plots to their study. Based on simple mean-field theory we have shown that the already observed zero-field first-order magnetic transition corresponds actually to a triple point where three first-order lines meet and that two of these first-order lines end in second-order critical points at finite fields. The resulting $H-T$ phase diagram corresponding to $\mathrm{La}_{2 / 3} \mathrm{Ca}_{1 / 3} \mathrm{MnO}_{3}$ is summarized in Fig. 14. At low temperatures we show in Fig. 14 the expected zero-field first-order line corresponding to the transition between symmetrical magnetization states $\pm M(T)$. Then the coupling between magnetization and volume induces a triple point at a temperature $T^{*}=260 \mathrm{~K}$. For larger temperatures, $T>T^{*}$, two field-dependent first-order lines $T^{\mathrm{FO}}(H)$ separate low- and high-magnetization states, $M_{1}$ and $M_{2}$. When the magnetization jump $\Delta M^{\mathrm{FO}}=M_{2}-M_{1}$ vanishes, the first-

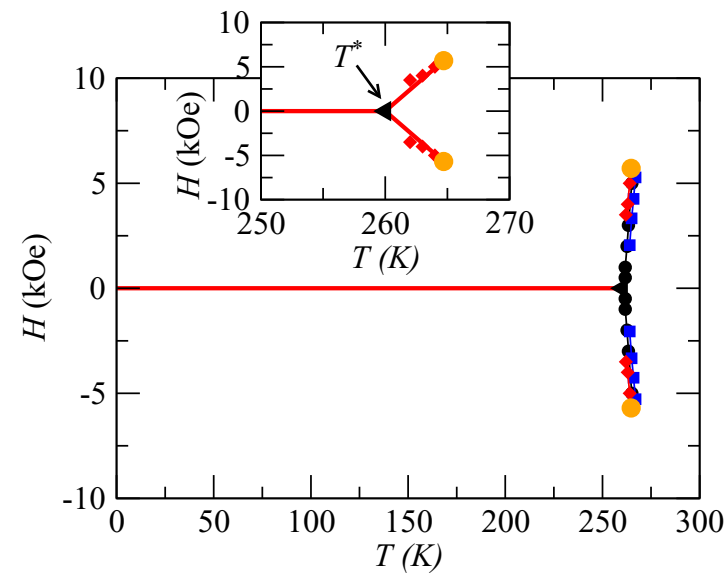

FIG. 14. $H-T$ phase diagram for $\mathrm{La}_{2 / 3} \mathrm{Ca}_{1 / 3} \mathrm{MnO}_{3}$. The typical first-order line at $H=0$ ends at $T^{*}=260 \mathrm{~K}$, which corresponds to a triple point where three first-order transition lines meet. Then, the two symmetrical first-order lines $T^{\mathrm{FO}}(H)$ end at the second-order critical points $\left(T_{c}, \pm H_{c}\right)=(264.7 \mathrm{~K}, \pm 5.7 \mathrm{Oe})$, indicated by the big orange dots. Black circles and blue squares correspond to the same data shown in Fig. 10, while red diamonds correspond to $H^{\mathrm{FO}}$ in Table I (below $T_{c}$ ). Note that for symmetry reasons the first-order line obtained for negative $H$ is a reflection of the one with positive $H$. The inset shows a zoom of the temperature region where the phase transitions take place, with $T^{*}$ indicated as a black triangle.

order lines end on second-order critical points located at $\left(T_{c}, \pm H_{c}\right)=(264.7 \mathrm{~K}, \pm 5.7 \mathrm{Oe})$. Due to the symmetry of the $M(H)$ curves, since $M(H)=-M(-H)$, the first-order line and critical point shown in Fig. 14 for negative $H$ values are the reflection of those we obtained for positive fields.

Although the formalism of Arrott plots was developed to extract information about second-order phase transitions, it is commonly used to indicate the presence of a first-order transition, taking as mere evidence the negative slope of the Arrott plot. However, we have stressed in this paper that if this were the case, the negative slope should increase (diminishing its absolute value) with increasing temperature, a fact that has not been experimentally observed. In fact, the negative slope is rather an artifact originated in drawing an Arrott plot close to the vicinity of a second-order critical point but with the incorrect order parameter $M$. Moreover, we have shown that when the modified Arrott plot is constructed using the symmetric order parameter $\Delta \phi$ the second-order character is recovered and the location of the critical point can be obtained.

It is also important to mention that once the volumemagnetization coupling is turned on, as in the mean-field model studied here, then a first-order jump in the volume of the system should accompany the first-order jump in the magnetization. This means that when the first-order line $\tau^{\mathrm{FO}}(h)$ is crossed, there exists a volume change $\Delta v^{\mathrm{FO}}=\eta K^{\prime}\left[\left(m_{2}^{\mathrm{FO}}\right)^{2}-\left(m_{1}^{\mathrm{FO}}\right)^{2}\right]$. Equivalently, a jump should be present in the linear thermal expansion, as already observed [39-41].

We would also like to stress that, although more elaborate models beyond the mean-field approach can provide information about magnetic correlations and susceptibility corrections, as shown in Ref. [26], the mean-field approximation presented 
here is enough to obtain second-order critical points at finite magnetic field and allows one to discuss the proper use of Arrott plots. A mean-field approach is arguably not the best to describe certain phase transition parameters, such as the actual values of the critical exponents determined experimentally. In this case a more accurate description can be obtained using Arrott-Noakes plots and more involved approaches.

Finally, we emphasize that our results are based on an expansion of the free energy close to $m=0$ and therefore the values obtained for $m_{c}, \tau_{c}$, and $h_{c}$ correspond to this free-energy expansion close to $m=0$. In addition, we have performed further expansions of the (already expanded) free-energy around $m=m_{c}$ to show how the Arrott plot emerges in the second-order critical point. However, though the expansion of the free energy around $m=0$ and the subsequent expansion around $m_{c}$ allow one to exploit all the simplicity of Landau-type arguments, the same physical properties are all contained in the full free-energy model within the molecular-field approximation, Eq. (A5). Therefore, the second-order critical point and the phase diagram presented here are also expected to be contained in more elaborate models considering a linear coupling between magnetization and specific volume or lattice distortions in general.

\section{ACKNOWLEDGMENTS}

We would like to thank $H$. Salva for providing the $\mathrm{La}_{2 / 3} \mathrm{Ca}_{1 / 3} \mathrm{MnO}_{3}$ sample and $\mathrm{C}$. Ramos for a critical reading of the manuscript. This work was partially supported by CONICET (Argentina) under Projects No. PIP11220120100250CO and No. PIP490-2012-2014, by ANPCyT (Argentina) under Project No. PICT-2011-0752, and by Universidad Nacional de Cuyo.

\section{APPENDIX A: MOLECULAR-FIELD FREE-ENERGY MODEL}

In order to justify the Landau free-energy model used in Sec. II, our starting point is the free-energy model originally developed 50 years ago by Rodbell and collaborators [16,25]. We shall consider as the free-energy density the free-energy per formula unit, which can be written as

$$
F[M, V]=U-T S-H M+P V,
$$

where $U$ and $S$ are the internal energy and entropy per formula unit, $T$ is the temperature, $H$ is the external magnetic field, and $P$ is the pressure. $M$ and $V$ are the magnetization and volume per formula unit. Within the molecular-field approximation for arbitrary spin $j$ and considering a constant isothermal compressibility $K$ the internal energy density becomes

$$
U[M, V]=-N k_{B} T_{c} \frac{3}{2} \frac{j}{j+1}\left(\frac{M}{M_{S}}\right)^{2}+\frac{1}{2 K} \frac{\left(V-V_{0}\right)^{2}}{V_{0}},
$$

with $N$ the number of magnetic ions per formula unit, and $M_{S}$ and $V_{0}$ the corresponding saturation magnetization and zero temperature volume. The temperature scale $T_{c}$ is proportional to the exchange interaction and corresponds to the Curie temperature of the pure magnetic system. The entropy density can be decomposed into a magnetic and lattice contribution, $S[M, V]=S_{j}[M]+S_{l}[V]$. According to Ref. [16] the magnetic contribution within the molecular-field approximation is

$$
\begin{aligned}
S_{j}[M]= & N k_{B}\left\{\ln 2-\frac{1}{2} \ln \left[1-\left(\frac{M}{M_{S}}\right)^{2}\right]\right. \\
& \left.-\frac{M}{M_{S}} \arctan \left(\frac{M}{M_{S}}\right)\right\} .
\end{aligned}
$$

Assuming a constant thermal expansion coefficient $\alpha$ and constant specific heat at constant volume $C_{V}$, the lattice entropy can be written as

$$
S_{l}[V]=C_{V} \ln T+\alpha \frac{V-V_{0}}{K} .
$$

Therefore, for a system with $j=1 / 2$ [42] the free energy becomes

$$
\begin{aligned}
F= & -\frac{N k_{B} T_{c}}{2}\left(\frac{M}{M_{\mathrm{S}}}\right)^{2}+\frac{1}{2 K} \frac{\left(V-V_{0}\right)^{2}}{V_{0}} \\
& -N k_{B} T\left\{\ln 2-\frac{1}{2} \ln \left[1-\left(\frac{M}{M_{\mathrm{S}}}\right)^{2}\right]\right. \\
& \left.-\left(\frac{M}{M_{\mathrm{S}}}\right) \arctan \left(\frac{M}{M_{\mathrm{S}}}\right)\right\} \\
& -T\left[C_{V} \ln T+\frac{\alpha}{K}\left(V-V_{0}\right)\right]-H M+P V .
\end{aligned}
$$

Up to this point the functional free energy can be split into two independent contributions, the magnetic and the lattice parts, i.e., $F=F_{0}+F_{M}+F_{V}\left(F_{0}\right.$ stands for the free-energy part which is both independent of $M$ and $V$ ). Expanding the magnetic entropy contribution around $M=0$ one obtains

$$
\begin{aligned}
F_{M}= & \frac{N k_{B}}{2}\left(T-T_{c}\right)\left(\frac{M}{M_{\mathrm{S}}}\right)^{2}+\frac{N k_{B} T}{12}\left(\frac{M}{M_{\mathrm{S}}}\right)^{4} \\
& +\frac{N k_{B} T}{30}\left(\frac{M}{M_{\mathrm{S}}}\right)^{6}+\cdots-H M,
\end{aligned}
$$

which gives the usual second-order magnetic transition at $T_{c}$ in a mean-field approximation. On the other hand, the volumedependent part becomes (using from now on $C_{V}=0$ )

$$
F_{V}=\frac{1}{2 K} \frac{\left(V-V_{0}\right)^{2}}{V_{0}}-\frac{T \alpha V}{K}+P V .
$$

The next step is to turn on the effective coupling between magnetic and volume contributions. This is achieved by considering the variation of the exchange interaction with volume through a variation of $T_{c}$ :

$$
\eta=\frac{V_{0}}{T_{0}} \frac{\partial T_{c}}{\partial V}
$$

with $T_{0}$ the Curie temperature when the coupling term is absent. The nondimensional parameter $\eta$ controls the coupling term. Within a linear approximation,

$$
T_{c}=T_{0}\left[1+\eta \frac{\left(V-V_{0}\right)}{V_{0}}\right] \text {. }
$$


Replacing this last expression in the free energy, the coupling term can be written as

$$
F_{M V}=-\eta \frac{N k_{B} T_{0}}{2} \frac{V-V_{0}}{V_{0}}\left(\frac{M}{M_{\mathrm{S}}}\right)^{2} .
$$

Finally, the different contributions to free-energy density $f=2 F /\left(N k_{B} T_{0}\right)=f_{0}+f_{m}+f_{v}+f_{m v}$ can be written in terms of the reduced magnetization and volume variables $m=M / M_{S}$ and $v=\left(V-V_{0}\right) / V_{0}$ as

$$
\begin{gathered}
f_{m}=(\tau-1) m^{2}+\frac{\tau}{6} m^{4}+\frac{\tau}{15} m^{6}-h m, \\
f_{v}=\frac{1}{2 K^{\prime}} v^{2}-\frac{\tau \alpha^{\prime}}{K^{\prime}} v+p v, \\
f_{m v}=-\eta v m^{2},
\end{gathered}
$$

with $h=2 H M_{S} /\left(N k_{B} T_{0}\right), K^{\prime}=K N k_{B} T_{0} /\left(2 V_{0}\right), \alpha^{\prime}=\alpha T_{0}$, and $p=2 P V_{0} /\left(N k_{B} T_{0}\right)$.

\section{APPENDIX B: SYMMETRIZED SECOND-ORDER TRANSITION}

It is instructive to analyze the physics around the secondorder critical points $\left(\tau_{c}, \pm h_{c}\right)$. In this case, as a first step, one can perform an expansion of the free energy in terms of the difference between the magnetization and its critical value $m_{c}$. We thus define $\phi=m-m_{c}$ as a small parameter and expand the free energy accordingly to obtain

$$
\tilde{f}=f\left(m_{c}, \tau_{c}, h_{c}\right)+\frac{1}{2} r \phi^{2}-w \phi^{3}+u \phi^{4}-\mu \phi,
$$

where $r, w, u$, and $\mu$ are in general temperature-dependent coefficients and which can be considered to depend linearly on $\Delta \tau=\tau-\tau_{c}$ close to the critical point. Then

$$
\begin{gathered}
r(\tau)=\left.\left.\frac{\partial^{2} f}{\partial m^{2}}\right|_{m_{c}} \approx \frac{\partial}{\partial \tau}\left(\left.\frac{\partial^{2} f}{\partial m^{2}}\right|_{m_{c}}\right)\right|_{\tau_{c}} \Delta \tau, \\
w(\tau)=-\left.\frac{1}{3 !} \frac{\partial^{3} f}{\partial m^{3}}\right|_{m_{c}} \approx-\left.\frac{1}{3 !} \frac{\partial}{\partial \tau}\left(\left.\frac{\partial^{3} f}{\partial m^{3}}\right|_{m_{c}}\right)\right|_{\tau_{c}} \Delta \tau, \\
u(\tau)=-\left.\frac{1}{4 !} \frac{\partial^{4} f}{\partial m^{4}}\right|_{m_{c}} \approx-\left.\frac{1}{4 !} \frac{\partial}{\partial \tau}\left(\left.\frac{\partial^{4} f}{\partial m^{4}}\right|_{m_{c}}\right)\right|_{\tau_{c}} \Delta \tau, \\
\mu(\tau)=-\left.\frac{\partial f}{\partial m}\right|_{m_{c}} \approx \Delta h-\left.\frac{\partial}{\partial \tau}\left(\left.\frac{\partial f}{\partial m}\right|_{m_{c}}\right)\right|_{\tau_{c}} \Delta \tau,
\end{gathered}
$$

where in the last expression an expansion in $\Delta h=h-h_{c}$ has also been considered. In particular, the effective field can be expressed as $\mu=\Delta h-\tilde{\mu} \Delta \tau$, with $\widetilde{\mu}=\left.\partial_{\tau m}^{2} f\right|_{m_{c}, \tau_{c}}$. At the critical point, by definition [first term in Eq. (B5)] one has $\mu=0$ and then

$$
h=h_{c}-\tilde{\mu}\left(\tau-\tau_{c}\right) .
$$

This defines the critical isochore in the vicinity of the critical point.

Using the definitions above, one has

$$
\begin{gathered}
\mu \approx \Delta h-\tilde{\mu} \Delta \tau ; \quad \tilde{\mu}=2 m_{c}\left(a+1 / 3 m_{c}^{2}+1 / 5 m_{c}^{4}\right), \\
r \approx \tilde{r} \Delta \tau ; \quad \tilde{r}=2\left(a+m_{c}^{2}+m_{c}^{4}\right),
\end{gathered}
$$

$$
\begin{gathered}
w \approx \tilde{w} \Delta \tau ; \quad \tilde{w}=2 m_{c}\left(1 / 3+2 / 3 m_{c}^{2}\right), \\
u \approx \tilde{u} \Delta \tau ; \quad \tilde{u}=1 / 6+m_{c}^{2} .
\end{gathered}
$$

Now, we can consider that the low- and high-magnetization states can be written as

$$
m^{1,2}=m_{c}+\phi_{0} \pm \Delta \phi,
$$

with $\Delta \phi$ a small symmetric quantity and $\phi_{0}$ defined below. Expanding now the free-energy density, Eq. (B1), close to the second-order critical point in terms of $\Delta \phi$ one can write

$$
\begin{aligned}
\tilde{f}= & f\left(m_{c}, \tau_{c}, h_{c}\right)-\mu\left(\phi_{0}\right) \Delta \phi+\frac{1}{2} r\left(\phi_{0}\right) \Delta \phi^{2} \\
& -w\left(\phi_{0}\right) \Delta \phi^{3}+u \Delta \phi^{4},
\end{aligned}
$$

where

$$
\begin{gathered}
\mu\left(\phi_{0}\right)=-\left.\frac{\partial \tilde{f}}{\partial \phi}\right|_{\phi_{0}}=\mu-r \phi_{0}+3 w \phi_{0}^{2}-4 u \phi_{0}^{3}, \\
r\left(\phi_{0}\right)=\left.\frac{\partial^{2} \tilde{f}}{\partial \phi^{2}}\right|_{\phi_{0}}=r-6 w \phi_{0}+12 u \phi_{0}^{2}, \\
w\left(\phi_{0}\right)=-\left.\frac{1}{3 !} \frac{\partial^{3} \tilde{f}}{\partial \phi^{3}}\right|_{\phi_{0}}=w-4 u \phi_{0}, \\
u\left(\phi_{0}\right)=\left.\frac{1}{4 !} \frac{\partial^{4} \tilde{f}}{\partial \phi^{4}}\right|_{\phi_{0}}=u .
\end{gathered}
$$

With the goal of having a symmetric free energy with respect to the order parameter, the value of $\phi_{0}$ is defined such that $w\left(\phi_{0}\right)=0$, thus eliminating the third-order term in the freeenergy expansion. Then,

$$
\phi_{0}=\frac{w}{4 u} \approx \frac{\widetilde{w} \Delta \tau}{4 u}=\widetilde{\phi}_{0} \Delta \tau
$$

where the linear approximation in $\Delta \tau$ has been used for $w$, together with a constant value for $u$ [to the order of our analysis, the temperature dependence of $u$ can be ignored and $u=(1 / 4 !)\left(\partial^{4} f / \partial m^{4}\right)_{m_{c}, \tau_{c}}$. The first-order term can also be eliminated using $\mu\left(\phi_{0}\right)=0$, which implies a temperature dependence for $\mu$,

$$
\mu=\frac{r w}{4 u}-\frac{w^{3}}{8 u^{2}} \approx \frac{\tilde{r} \widetilde{w}}{4 u} \Delta \tau^{2}+\mathcal{O}\left(\Delta \tau^{3}\right) .
$$

The free energy Eq. (B12) can now be written as

$$
\tilde{f}=f_{0}\left(\phi_{0}\right)+\frac{1}{2} r\left(\phi_{0}\right) \Delta \phi^{2}+u \Delta \phi^{4},
$$

where, using the definition of $\phi_{0}$,

$$
r\left(\phi_{0}\right)=r-\frac{3 w^{2}}{4 u} \approx \tilde{r} \Delta \tau-\frac{3 \tilde{w}}{4 u} \Delta \tau^{2} \approx \tilde{r} \Delta \tau .
$$

Therefore, finally, the free energy becomes

$$
\tilde{f}=f_{0}\left(\phi_{0}\right)+\frac{1}{2} \widetilde{r}\left(\tau-\tau_{c}\right) \Delta \phi^{2}+u \Delta \phi^{4},
$$

which is, as expected, the standard free-energy form for a second-order phase transition with an order parameter $\Delta \phi$ such that

$$
\Delta \phi=\left\{\begin{array}{lll}
0 & \text { for } & \tau>\tau_{c}, \\
\pm \sqrt{-\frac{\widetilde{r}\left(\tau-\tau_{c}\right)}{4 u}} & \text { for } & \tau<\tau_{c} .
\end{array}\right.
$$


Since this is a second-order transition, the Arrott plot in terms of the variables $(\mu, \Delta \phi)$ is well behaved when plotting $\mu / \Delta \phi$ against $\Delta \phi^{2}$ since

$$
\frac{\mu}{\Delta \phi}=\tilde{r} \Delta \tau+4 u \Delta \phi^{2} .
$$

The next step is to use this well behaved Arrott plot to construct the correct Arrott plot close to the second-order critical point using the original variables $(h, m)$. Recalling that $\mu=\Delta h-\tilde{\mu} \Delta \tau$ and $\Delta \phi=m-m_{c}-\phi_{0}$ the Arrott relation becomes

$$
\frac{\Delta h-\tilde{\mu} \Delta \tau}{m-m_{c}-\tilde{w} \Delta \tau /(4 u)}=\tilde{r} \Delta \tau+4 u\left(m-m_{c}-\tilde{w} \Delta \tau /(4 u)\right)^{2} .
$$

[1] K. Huang, Statistical Mechanics (John Wiley \& Sons, New York, 1987).

[2] J. M. D. Coey, Magnetism and Magnetic Materials (Cambridge University Press, Cambridge, UK, 2009).

[3] H. E. Stanley, Introduction to Phase Transitions and Critical Phenomena (Clarendon Press, Oxford, 1971).

[4] A. Arrott, Phys. Rev. 108, 1394 (1957).

[5] A. Arrott and J. E. Noakes, Phys. Rev. Lett. 19, 786 (1967).

[6] Y. S. Hor, P. Roushan, H. Beidenkopf, J. Seo, D. Qu, J. G. Checkelsky, L. A. Wray, D. Hsieh, Y. Xia, S.-Y. Xu, D. Qian, M. Z. Hasan, N. P. Ong, A. Yazdani, and R. J. Cava, Phys. Rev. B 81, 195203 (2010).

[7] J. Mira, J. Rivas, M. Vázquez, J. M. García-Beneytez, J. Arcas, R. D. Sánchez, and M. A. Señarís-Rodríguez, Phys. Rev. B 59, 123 (1999).

[8] H. Ohno, D. Chiba, F. Matsukura, T. Omiya, E. Abe, T. Dietl, Y. Ohno, and K. Ohtani, Nature (London) 408, 944 (2000).

[9] C. S. Hong, W. S. Kim, and N. H. Hur, Phys. Rev. B 63, 092504 (2001).

[10] H. Ohno, Science 281, 951 (1998).

[11] R. L. Hadimani, Y. Melikhov, J. E. Snyder, and D. C. Jiles, J. Appl. Phys. 103, 033906 (2008).

[12] N. T. Huy, A. Gasparini, D. E. de Nijs, Y. Huang, J. C. P. Klaasse, T. Gortenmulder, A. de Visser, A. Hamann, T. Görlach, and H. v. Löhneysen, Phys. Rev. Lett. 99, 067006 (2007).

[13] L. Zhang, J. Fan, L. Li, R. Li, L. Ling, Z. Qu, W. Tong, S. Tan, and Y. Zhang, Europhys. Lett. 91, 57001 (2010).

[14] M. Phan, G. Woods, A. Chaturvedi, S. Stefanoski, G. Nolas, and H. Srikanth, Appl. Phys. Lett. 93, 252505 (2008).

[15] B. K. Banerjee, Phys. Lett. 12, 16 (1964).

[16] C. P. Bean and D. S. Rodbell, Phys. Rev. 126, 104 (1962).

[17] J. Mira, J. Rivas, F. Rivadulla, and M. L. Quintela, Physica B: Condens. Matter 320, 23 (2002).

[18] J. E. Gordon, C. Marcenat, J. P. Franck, I. Isaac, G. Zhang, R. Lortz, C. Meingast, F. Bouquet, R. A. Fisher, and N. E. Phillips, Phys. Rev. B 65, 024441 (2001).

[19] A. Fujita, Y. Akamatsu, and K. Fukamichi, J. Appl. Phys. 85, 4756 (1999).

[20] D. P. Rojas, J. I. Espeso, J. Rodríguez Fernández, J. C. Gómez Sal, J. Sanchez Marcos, and H. Müller, Phys. Rev. B 80, 184413 (2009).

[21] P. Velasco, J. Mira, F. Guinea, J. Rivas, M. J. Martínez-Lope, J. A. Alonso, and J. L. Martínez, Phys. Rev. B 66, 104412 (2002).

[22] M. Parra-Borderías, F. Bartolomé, J. Herrero-Albillos, and L. M. García, J. Alloys Compd. 481, 48 (2009).

[23] L. A. Burrola-Gándara, C. R. Santillan-Rodriguez, F. J. Rivera-Gomez, R. J. Saenz-Hernandez, M. E. Botello-Zubiate, and J. A. Matutes-Aquino, J. Appl. Phys. 117, 17D144 (2015).

[24] Z. Qu, L. Ling, L. Zhang, L. Pi, and Y. Zhang, Solid State Commun. 151, 917 (2011).

[25] R. W. De Blois and D. S. Rodbell, Phys. Rev. 130, 1347 (1963).

[26] H. Yamada, Phys. Rev. B 47, 11211 (1993).

[27] L. Lundgren, G. Tarmohamed, O. Beckman, B. Carlsson, and S. Rundqvist, Phys. Scr. 17, 39 (1978).

[28] O. Tegus, G. Lin, W. Dagula, B. Fuquan, L. Zhang, E. Brück, F. De Boer, and K. Buschow, J. Magn. Magn. Mater. 290-291, 658 (2005).

[29] P. von Ranke, N. De Oliveira, and S. Gama, J. Magn. Magn. Mater. 277, 78 (2004).

[30] S. Yang, X. Ren, and X. Song, Phys. Rev. B 78, 174427 (2008).

[31] P. Sarkar, S. Arumugam, P. Mandal, A. Murugeswari, R. Thiyagarajan, S. E. Muthu, D. M. Radheep, C. Ganguli, K. Matsubayshi, and Y. Uwatoko, Phys. Rev. Lett. 103, 057205 (2009).

[32] Note that for simplicity we are using $m$ to denote the location of the minimum of the free-energy density. Therefore, the magnetization $m$ in the equation of state $m(h, \tau)$ corresponds to the location of the minimum of the free-energy density $f(m, \tau, h)$.

[33] Q. Huang, A. Santoro, J. W. Lynn, R. W. Erwin, J. A. Borchers, J. L. Peng, K. Ghosh, and R. L. Greene, Phys. Rev. B 58, 2684 (1998).

[34] J. Mira, J. Rivas, F. Rivadulla, C. Vázquez-Vázquez, and M. A. López-Quintela, Phys. Rev. B 60, 2998 (1999).

[35] G.-m. Zhao, M. B. Hunt, and H. Keller, Phys. Rev. Lett. 78, 955 (1997).

[36] M. J. Calderón, A. J. Millis, and K. H. Ahn, Phys. Rev. B 68, 100401 (2003).

[37] D. Kim, B. Revaz, B. L. Zink, F. Hellman, J. J. Rhyne, and J. F. Mitchell, Phys. Rev. Lett. 89, 227202 (2002).

[38] F. Rivadulla, J. Rivas, and J. B. Goodenough, Phys. Rev. B 70, 172410 (2004).

[39] H. Salva, C. Ramos, A. Ghilarducci, R. Sánchez, and C. Vázquez-Vázquez, J. Magn. Magn. Mater. 226-230, 590 (2001).

[40] J. Mira, J. Rivas, L. E. Hueso, F. Rivadulla, M. A. López Quintela, M. A. Señarís Rodríguez, and C. A. Ramos, Phys. Rev. B 65, 024418 (2001).

[41] F. Rivadulla, M. Otero-Leal, A. Espinosa, A. de Andrés, C. Ramos, J. Rivas, and J. B. Goodenough, Phys. Rev. Lett. 96, 016402 (2006).

[42] Although we have used here $j=1 / 2$, a general value of the spin $j$ can be recovered by replacing the Curie temperature $T_{c}$ by $3 T_{c} j /(j+1)$. 